\title{
Lysosomal storage disease overview
}

\author{
Angela Sun \\ Department of Pediatrics, Seattle Children's Hospital, University of Washington, Seattle, WA, USA \\ Correspondence to: Angela Sun, MD. Seattle Children's Hospital, University of Washington, 4800 Sand Point Way NE, Seattle, WA 98105, USA. \\ Email: angela.sun@seattlechildrens.org.
}

\begin{abstract}
The lysosomal storage diseases (LSDs) are a group of inherited metabolic disorders that are caused for the most part by enzyme deficiencies within the lysosome resulting in accumulation of undegraded substrate. This storage process leads to a broad spectrum of clinical manifestations depending on the specific substrate and site of accumulation. Examples of LSDs include the mucopolysaccharidoses, mucolipidoses, oligosaccharidoses, Pompe disease, Gaucher disease, Fabry disease, the Niemann-Pick disorders, and neuronal ceroid lipofuscinoses. This review summarizes the main clinical features, diagnosis, and management of LSDs with an emphasis on those for which treatment is available.
\end{abstract}

Keywords: Lysosomal storage disease (LSD); mucopolysaccharidosis (MPS); Gaucher; Fabry; Niemann-Pick

Submitted Oct 01, 2018. Accepted for publication Oct 17, 2018.

doi: 10.21037/atm.2018.11.39

View this article at: http://dx.doi.org/10.21037/atm.2018.11.39

\section{Introduction}

The lysosomal storage diseases (LSDs) are a group of inherited metabolic disorders that are caused for the most part by enzyme deficiencies within the lysosome resulting in accumulation of undegraded substrate. This storage process leads to a broad spectrum of clinical manifestations depending on the specific substrate and site of accumulation. On the whole, these disorders are progressive, although the rate of progression is variable, and the advent of novel therapies has changed the natural history of the disease in some cases. This review will provide an overview of LSDs with a particular focus on those for which treatment is available.

\section{Mucopolysaccharidoses}

Mucopolysaccharides, also known as glycosaminoglycans, are complex sugar molecules found in connective and other tissues throughout the body such as skin, cartilage, cornea, liver, spleen, and vascular tissue. Examples of mucopolysaccharides include dermatan sulfate, heparan sulphate, keratan sulphate, chondroitin sulphate, and hyaluronic acid. Their degradation requires a variety of enzymes (Table 1). All mucopolysaccharidosis (MPS) disorders are inherited in an autosomal recessive manner with the exception of MPS type II, which is X-linked.

\section{MPS I and MPS II}

MPS type I, historically known as Hurler or Scheie syndrome depending on clinical severity, and MPS type II, also known as Hunter syndrome, are the most common types of MPS. Both have a range of clinical severity. The classic, severe presentation is a pre-school age child with developmental delay, short stature, recurrent ear and respiratory infections, hepatosplenomegaly, and coarsening of facial features. Over time, the child develops hearing loss, cardiac valve disease, airway obstruction, skeletal contractures, and distinctive facial appearance with macrocephaly, thick eyebrows, gingival hypertrophy, macroglossia, and thickening of the lips and nasal alae. Intellect is impaired, and patients undergo regression as the disease progresses. IQ is $<70$ in $61 \%$ of patients who are untreated and borderline [70-85] in $25 \%$ at 2-3 years (1). Other complications include corneal clouding, carpal tunnel syndrome, hydrocephalus, glaucoma, cardiac arrhythmias, cervical instability, and spinal cord compression. The characteristic radiographic findings, collectively termed dysostosis multiplex, include J-shaped sella turcica, 
Table 1 The mucopolysaccharidoses, associated genes and enzymes

\begin{tabular}{lll}
\hline Disorder & Gene & Enzyme deficiency \\
\hline MPS I (Hurler, Scheie syndrome) & IDUA & Alpha-L-iduronidase \\
MPS II (Hunter syndrome) & IDS & Iduronate-2-sulfatase \\
MPS IIIA (Sanfilippo syndrome) & SGSH & Heparan-N-sulfatase \\
MPS IIIB (Sanfilippo syndrome) & NAGLU & N-acetylglucosaminidase \\
MPS IIIC (Sanfilippo syndrome) & HGSNAT & Acetyl CoA glucosamine N-acetyltransferase \\
MPS IIID (Sanfilippo syndrome) & GNS & N-acetyl-glucosamine-6-sulfatase \\
MPS IVA (Morquio syndrome) & GALNS & N-acetylgalactosamine-6-sulfate sulfatase \\
MPS IVB (Morquio syndrome) & GLB1 & $\beta$-galactosidase \\
MPS VI (Maroteaux-Lamy syndrome) & ARSB & Arylsulfatase B \\
MPS VII (Sly syndrome) & GUSB & $\beta$-glucuronidase \\
MPS IX & HYAL1 & Hyaluronidase \\
\hline
\end{tabular}

oar shaped ribs, pointing of the proximal metacarpals and metatarsals, poorly developed acetabulum, etc. (2). Untreated, the life expectancy is the second or third decade.

Patients with the milder forms of MPS I and II have less prominent somatic findings and no central nervous system (CNS) disease. Intellect may be normal.

Quantitative urine mucopolysaccharide analysis is the first test in screening a patient with suspected MPS I or II. If urine mucopolysaccharide excretion is elevated, blood should be sent for enzyme activity as either a single assay or panel. The latter can be helpful when the features of the patient do not allow for a specific diagnosis. Decreased enzyme activity confirms the diagnosis. Genetic testing should also be done as genotype-phenotype correlations can aid in prognostication (3). Radiographs showing dysostosis multiplex support the diagnosis.

The mainstay of treatment is enzyme replacement therapy (ERT) with laronidase (Aldurazyme ${ }^{\circledR}$ ) for MPS I and idursulfase (Elaprase ${ }^{\circledR}$ ) for MPS II. ERT slows the progression of disease but is not curative. The protein does not cross the blood brain barrier, thus, the neurologic disease remains untreated. Hematopoietic stem cell transplantation (HSCT) can be performed in MPS I patients who are less than 2 years old and stabilizes neurocognitive function (4) due to the repopulation of microglial cells and restoration of enzyme activity in the brain (5). The rationale for early transplant is that disease manifestations are less severe in younger patients, and transplant outcomes are therefore better (4). Patients with severe MPS I who undergo bone marrow transplantation have a mean IQ of 76 across all age groups (1). HSCT was initially not successful for MPS II patients (6), possibly due to severe baseline disease in some patients, but more recent experiences indicate benefit beyond ERT alone and stabilization of neurocognitive function $(7,8)$.

In addition to ERT and HSCT, patients with MPS I and II require management by multiple specialists for their many medical complications. Early in life, upper airway obstruction often necessitates tonsilloadenoidectomy. Cardiac function must be monitored regularly. MRI to evaluate for cervical instability and spinal stenosis should be performed at least biannually. Clinical management guidelines for MPS I and MPS II have been published $(9,10)$.

\section{MPS III}

There are 4 subtypes of MPS type III (Table 1), with type IIIA being the most common. All are due to defects in heparan sulphate metabolism and result in the same clinical phenotype. MPS III can be differentiated from the other MPS disorders by the predominance of neuropsychiatric rather than somatic problems. Children manifest with developmental delay and intellectual disability early on. In severe cases, development plateaus between 36-40 months, and these patients never learn to speak more than a few single words $(11,12)$. Patients with less severe disease may continue to acquire skills until 5-10 years of age. As the disease progresses, affected children develop significant behavioral problems including aggression, hyperactivity, decreased attention span, tantrums, anxiety, and destructive 
behaviors. Sleep disturbance is common with both difficulty falling asleep and frequent nighttime wakening (11). This stage can be very difficult to manage, and medication may be necessary to control behaviors and aid sleep $(13,14)$. Over time, progressive MPS deposition in the brain leads to seizures in $60-70 \%$, spasticity, and feeding difficulties. Those with severe disease undergo rapid regression with loss of 9-15 developmental quotient (DQ) points per year (12). In more mildly affected individuals, the rate of regression is slower, with a loss of 3.7 DQ points of per year. Patients gradually become encephalopathic with loss of language and ambulation culminating in a vegetative state.

Somatic problems are mild in MPS III. Hepatosplenomegaly is often present but not usually diagnosed clinically. Cardiac valve thickening may occur; otherwise cardiac abnormalities are rare. Subtle features of dysostosis multiplex have been reported. Facial coarsening is mild or absent.

Diagnosis of MPS III is based on demonstration of elevated urinary excretion of mucopolysaccharides, enzyme deficiency, and genetic testing. There is no treatment available for MPS III. Clinical trials have been conducted to investigate various methods of delivering ERT into the CNS $(15,16)$. Age of death is generally in the teenage years, with pneumonia being the most common cause (11).

\section{MPS IV}

MPS type IV (Morquio syndrome) is caused by one of 2 enzyme deficiencies (Table 1), both of which result in a skeletal dysplasia. MPS IVA and IVB are difficult to distinguish clinically, but the former is far more common. Age of onset is generally in the first few years of life. Clinical manifestations include short stature, pectus carinatum, forearm deformity, genu valgum, scoliosis, hip dysplasia, odontoid hypoplasia with atlantoaxial instability, and dental abnormalities. Non-skeletal features include corneal clouding, hearing loss, obstructive or restrictive lung disease, and cardiac dysfunction. Intellect is normal. At 18 years, mean height in males is $122.4 \pm 21.5 \mathrm{~cm}$ and in females is $113.1 \pm 22.6 \mathrm{~cm}$ (17). However, as with other MPS disorders, a considerable spectrum of clinical severity exists. Mildly affected individuals may attain normal adult height and have few clinical complications (18).

Diagnosis of MPS IV can be complicated by the fact that urine mucopolysaccharide quantitation may be falsely normal, especially in older patients (19). Enzymatic and genetic testing should be pursued if clinical suspicion is high. Radiographs showing dysostosis multiplex support the diagnosis.
ERT is available for MPS IVA but not MPS IVB. Elosulfase alfa $\left(\right.$ VIMIZIM $\left.^{\circledR}\right)$ reduces keratan sulfate accumulation and improves endurance as assessed by a 6-minute walk test (6MWT) (20). Modest improvements are seen in pulmonary function testing. Patients require followup by numerous specialists including Biochemical Genetics, Orthopedics, Otolaryngology, Neurology, Cardiology, Pulmonary, Rehabilitation Medicine, Ophthalmology, Audiology, and Dental. Comprehensive management guidelines for MPS IVA have been published (19).

\section{MPS VI}

MPS VI is rare with an estimated incidence of 0.36 to 1.30 per 100,000 live births (21). Like MPS types I and II, it is characterized by storage of dermatan sulphate, but unlike the other two, heparan sulphate metabolism is not impaired. Therefore, intellect is generally normal. MPS VI otherwise shares many of the features of MPS I and II including short stature, coarse facies, corneal clouding, airway obstruction, cardiac valve abnormalities, hepatosplenomegaly, inguinal and umbilical hernias, dysostosis multiplex, joint contractures, carpal tunnel syndrome, and hip dysplasia. The age of onset and rate of disease progression are variable. Diagnosis is based on increased urinary excretion of mucopolysaccharides, low arylsulfatase B enzyme activity in leukocytes, and the identification of pathogenic variants in $A R S B$. ERT is available for MPS VI. Galsulfase $\left(\right.$ Naglazyme $^{\circledR}$ ) improves growth in patients with MPS VI, (22), reduces cardiac hypertrophy, prevents progression of cardiac valve disease (23), and preserves joint range of motion (24). Treatment must be started at a young age for maximal benefit. Comprehensive management guidelines are available for MPS VI (25).

\section{MPS VII}

MPS VII is an ultra-rare LSD with an estimated incidence of $0.05-0.29$ per 100,000 live births (21). $40-45 \%$ of patients with MPS VII present with nonimmune fetal hydrops (26,27). Fetal hydrops can occur in the other MPS disorders, though far less frequently, making it a relatively suggestive finding for MPS VII. In patients who survive beyond the neonatal period, intellectual disability is present in $86 \%$, coarse facies in $87 \%$, dysostosis multiplex in $90 \%$, joint contractures in $84 \%$, hepatosplenomegaly in $75 \%$, scoliosis in $69 \%$, corneal clouding in $63 \%$, obstructive airway disease in $44 \%$, cardiac valve disease in $50 \%$, and 
Table 2 The mucolipidoses and oligosaccharidoses, associated genes and enzymes

\begin{tabular}{lll}
\hline Disease & Gene & Encoded protein \\
\hline Mucolipidosis I (Sialidosis) & NEU1 & $\alpha$-neuraminidase \\
Mucolipidosis II/III & GNPTAB, GNPTG & UDP-N-acetylglucosamine- 1-phosphotransferase \\
Mucolipidosis IV & MCOLN1 & Mucolipin-1 \\
$\alpha$-mannosidosis & MAN2B1 & $\alpha$-mannosidase \\
$\beta$-mannosidosis & MANBA & $\beta$-mannosidase \\
Fucosidosis & FUCA1 & Fucosidase \\
Galactosialidosis & CTSA & Cathepsin A \\
Salla disease & SLC17A5 & Sialin \\
Infantile sialic acid storage disease & SLC17A5 & Sialin \\
Aspartylglucosaminuria & AGA & Aspartylglucosaminidase \\
Schindler disease & NAGA & $\alpha$-N-acetylgalactosaminidase \\
\hline
\end{tabular}

cardiomyopathy in $37 \%$ (26). Many of the other somatic problems of MPS I are also seen in MPS VII. The diagnosis of MPS VII can be made by urine mucopolysaccharide quantitation, measurement of $\beta$-glucuronidase activity in blood, and genetic testing. The mainstay of treatment is ERT. In clinical trials, vestronidase alfa (MEPSEVII ${ }^{\mathrm{TM}}$ ) was shown to improve various measures such a 6-minute walk test, gross and fine motor function, and fatigue, although the number of participants was small due to the rarity of the disorder.

\section{Mucolipidoses and oligosaccharidoses}

The mucolipidoses and oligosaccharidosis are relatively rare and are summarized in Table 2. They share many features with the MPS disorders including developmental delay, organomegaly, and dysostosis multiplex. All are progressive, and currently no disease-specific therapies are available for these conditions.

\section{Pompe disease}

Pompe disease is a disorder of glycogenolysis resulting in glycogen accumulation in lysosomes. Rupture of engorged lysosomes leads to tissue damage primarily involving skeletal and cardiac muscle. Patients with infantile-onset Pompe disease (IOPD) present in the first few months of life with weakness, hypotonia, developmental delay, feeding difficulties, failure to thrive, macroglossia, and hypertrophic cardiomyopathy. Prior to availability of ERT, infants with IOPD died in the first year of life from heart failure (28). Survivors often require respiratory support including mechanical ventilation, feeding tubes, walker and/ or wheelchairs. Individuals with late-onset Pompe disease (LOPD) have symptom onset anywhere from childhood to adulthood. Generally speaking, the earlier the age of onset, the more severe the disease and the faster the rate of progression. Individuals with adult-onset disease have normal heart function and may only complain of proximal muscle weakness.

The diagnosis of Pompe disease can be made based upon clinical presentation, measurement of alpha-glucosidase enzyme activity in blood, and molecular testing showing two pathogenic variants in the $G A A$ gene. Creatine kinase is universally elevated in patients with IOPD, and elevated urine hexose tetrasaccharide (Hex4) also supports the diagnosis.

ERT with alglucosidase alfa $\left(\right.$ Myozyme ${ }^{\circledR}$, Lumizyme $^{\circledR}$ ) has changed the natural history of Pompe disease. ERT effectively treats hypertrophic cardiomyopathy in IOPD, with improvement and/or normalization of left ventricular mass index and other cardiac parameters sometimes within weeks of starting therapy (29-31). Arrhythmias occur in a minority of patients $(29,32)$. IOPD has evolved from a fatal cardiac disease into a chronic neuromuscular disorder as skeletal muscle does not respond well to ERT. Ninety one percent of IOPD patients treated with ERT have weakness and hypotonia, $91 \%$ have dysarthria, $45 \%$ have dysphagia, 
$36 \%$ have feeding tube dependence, $36 \%$ require assistive mobility devices, and many need oxygen, BiPAP, or mechanical ventilation for respiratory insufficiency and obstructive sleep apnea (33). In patients with LOPD, ERT stabilizes respiratory capacity, improves motor function, maintains ambulation, and increases survival (34). Because of ongoing medical issues, patients with Pompe disease require multidisciplinary care. Clinical management guidelines are available for IOPD and LOPD (35-38).

\section{Disorders of sphingolipid metabolism}

\section{Gaucher disease}

Gaucher disease is the most common LSD with a prevalence of roughly 1/40,000 (39). In the Ashkenazi Jewish population, the prevalence was historically as high as 1/1,000 (39). Gaucher disease is caused by mutations in $G B A$ resulting in deficiency of glucocerebrosidase. Accumulation of glucosylceramide in the lysosomes of macrophages affects cells of the reticuloendothelial system, including liver, spleen, and bone marrow.

There are three clinical phenotypes: Gaucher disease type I, which does not have neurologic involvement, and types II and III, the so-called neuronopathic forms which both feature neurologic impairment. Gaucher disease type I accounts for more than $90 \%$ of cases of Gaucher disease $(40,41)$. Common disease manifestations include hepatosplenomegaly, thrombocytopenia, anemia, bone pain, and poor growth in children. With regard to bone disease specifically, $33 \%$ of patients suffer bone crises, $42 \%$ osteopenia, $40 \%$ marrow infiltration with Erlenmeyer flask deformity of the femur, $25 \%$ avascular necrosis, $15 \%$ pathologic fractures, and 8\% lytic lesions (40). Age of onset is highly variable and ranges from childhood to the seventh decade, but $2 / 3$ of patients are diagnosed before age 20 (40). The clinical variability can be explained in part by specific GBA mutations. The p.N370S allele, when homozygous or compound heterozygous, always results in type I disease (40). Individuals who are homozygous for the p.L444P allele most often have type II or III disease. Other genotypephenotype correlations have been reported $(42,43)$. The p.N370S and p.L444P alleles are common in the Ashkenazi Jewish population.

Gaucher disease type II is characterized by severe developmental delay, cachexia, seizures, horizontal gaze palsy, spasticity, neck retroflexion, opisthotonus, cortical thumbs, and other pyramidal signs. Some newborns present with fetal hydrops or congenital ichthyosis. Infants rapidly progress to total disability and death by 2 years.

Gaucher disease type III has a more insidious course. Other features in addition to those above include oculomotor apraxia, esotropia, and hearing loss. The somatic problems of Gaucher type I are often present but less severe. Children undergo slow cognitive regression in adolescence or early adulthood.

Gaucher disease can be diagnosed by measurement of glucocerebrosidase enzyme activity in leukocytes and identification of two pathogenic variants in the GBA gene. In some cases, bone marrow biopsy is performed and reveals the characteristic Gaucher cells with crumpled tissue paper appearance.

The treatment of Gaucher disease types I and III consists of either ERT or substrate reduction therapy (SRT). There are 3 FDA-approved ERT's, imiglucerase (Cerezyme $\left.^{\circledR}\right)$, velaglucerase alfa $\left(\mathrm{VPRIV}^{\circledR}\right)$, and taliglucerase alfa $\left(\right.$ Elelyso $\left.{ }^{\circledR}\right)$, and 2 FDA-approved SRT's, miglustat $\left(\right.$ Zavesca $\left.^{\circledR}\right)$ and eliglustat $\left(\right.$ Cerdelga $\left.^{\circledR}\right)$. SRT works by inhibiting the enzyme glucosylceramide synthase, the first step in glycosphingolipid synthesis, thereby limiting glucosylceramide production. One advantage of SRT is that it is administered orally rather than intravenously. Clinical practice varies widely, but most newly diagnosed patients are started on ERT initially. Some transition to SRT at a later age. Children with Gaucher disease should be started on ERT as soon as they are symptomatic $(44,45)$. Taliglucerase alfa, miglustat, and eliglustat are only approved for use in adults. Both ERT and SRT increase platelet count and hemoglobin concentration, decrease liver and spleen volume, and reduce Gaucher disease biomarkers such as chitotriosidase (46-52).

Abdominal magnetic resonance imaging (MRI) should be performed at regular intervals to monitor liver and spleen volumes. Historically, splenectomy was performed for patients with significant splenomegaly and thrombocytopenia, but ERT has obviated the need for this. The extent of bone marrow infiltration, osteosclerosis, and osteonecrosis can be assessed by MRI of the spine and femur, and bone mineral density by (dual-energy X-ray absorption) DEXA scan. Acute bone crises can be refractory to narcotics, but high dose steroids may relieve pain (53). Therapeutic goals and management guidelines are available for Gaucher disease type I $(44,45,54,55)$.

There is currently no treatment available for Gaucher disease type II. ERT is not recommended $(43,45)$. Care is supportive, with attention to issues common to other 
neurodegenerative conditions such nutrition, risk of aspiration, need for respiratory support, etc.

Less data on treatment efficacy are available for Gaucher disease type III. ERT and SRT improve hematologic and visceral parameters but do not appear to impact the neurologic disease $(56,57)$.

\section{Fabry disease}

Fabry disease is an X-linked LSD caused by mutations in $G L A$. Deficiency of the enzyme alpha-galactosidase results in accumulation of globotriaosylceramide (GL3) in tissues throughout the body and multi-organ system disease. Age of onset is highly variable and can range from early childhood to the fifth decade or later $(58,59)$. Some mutations are known to cause classic disease while others results in milder, late onset disease (60).

In classic males, the earliest symptoms are neuropathic pain, often in the form of acroparesthesias (burning, tingling pain crises in the hands and feet), and GI symptoms such as abdominal pain and diarrhea beginning at $5-10$ years of age (58). Angiokeratomas (clusters of purplish, non-blanching punctate lesions) and hypohidrosis may also present in childhood. Renal function progressively deteriorates as GL3 is deposited in the kidneys, and the average age of onset of clinical nephropathy is 27 years (61). Untreated, end stage renal disease develops at a median age of 47 years with a range of 21-56 years (62). Cardiac manifestations include left ventricular hypertrophy (LVH), arrhythmias, myocardial fibrosis, syncope, and exercise intolerance $(59,63)$. The age of onset of LVH is in the fourth to fifth decade. GL3 accumulation in cerebrovascular tissue leads to transient ischemic attacks (TIA) and stroke in $12 \%$ of males with mean age of onset 28.8 years for a cerebrovascular event (59). Other problems include tinnitus, hearing loss, a specific corneal opacity termed cornea verticillata, mood disorders including anxiety and depression, and an obstructive-type lung disease. Prior to the availability of ERT, the mean age of death in males was 45.5 years with renal failure being the most common cause (59).

Although Fabry disease is X-linked, rarely are affected females truly asymptomatic. In fact, some females have symptoms and disease burden on par with that of males. $4-25 \%$ of females with Fabry disease have cerebrovascular disease, $43-77 \%$ experience neuropathic pain, 18-26\% have LVH, and $35-39 \%$ have proteinuria $(64,65)$. The clinical variability in females can be explained by skewing of $\mathrm{X}$-inactivation, which is the normal process by which one of the $\mathrm{X}$ chromosomes in the female embryo is inactivated. If a disproportionate number of $\mathrm{X}$ chromosomes carrying the normal GLA allele are inactivated, then the individual will be more likely to manifest symptoms.

Fabry disease is diagnosed by enzyme assay showing deficiency of alpha-galactosidase activity and by genetic testing. In females, enzyme activity cannot be relied upon; genetic testing should be done to rule out or confirm the diagnosis. Elevated GL3 in plasma and urine is helpful in both initial diagnosis and long-term disease monitoring.

The mainstay of therapy for Fabry disease is ERT. In the United States, agalsidase beta (Fabrazyme ${ }^{\circledR}$ ) is the only approved ERT. Agalsidase alfa (Replagal ${ }^{\circledR}$ ) is commercially available in other countries. Despite clearance of GL3 on ERT, renal function continues to decline and does so more rapidly in patients with more severe baseline disease. In male patients with GFR $>60 \mathrm{~mL} / \mathrm{min} / 1.73 \mathrm{~m}^{2}$, the rate of decline was similar in treated and untreated groups (66). However, in those with GFR $<60 \mathrm{~mL} / \mathrm{min} / 1.73 \mathrm{~m}^{2}$, the mean decline in the treated group was less than the untreated group $\left(-3.04\right.$ compared to $\left.-6.80 \mathrm{~mL} / \mathrm{min} / 1.73 \mathrm{~m}^{2}\right)$. For male patients with LVH, ERT stabilizes left ventricular mass, although slight increases can be seen (66). Good data are lacking as to the effect of ERT on cerebrovascular disease (66), although one study did not find a decreased risk of TIA or stroke on ERT (67).

As far as initiation of therapy, it is recommended to start ERT in any symptomatic male or female patient regardless of age $(63,68)$. In asymptomatic male children with classical Fabry mutations, it is strongly recommended to consider ERT initiation by $8-10$ years. Asymptomatic female patients should be monitored closely for evidence of end organ involvement (e.g., microalbuminuria, recurrent abdominal pain, acroparesthesias, etc.).

In addition to ERT, patients with Fabry disease may require several adjunctive therapies to treat complications. For acroparesthesias, gabapentin and carbamazepine are typically prescribed. Opioid analgesics should be avoided. Patients with microalbuminuria should be started on an angiotensin converting enzyme inhibitor (ACEI) or angiotensin receptor blocker (ARB). Those who have experienced TIA or stroke should be placed on aspirin or clopidogrel. Lifestyle modifications may also improve symptom management and quality of life, such as avoiding extreme temperatures, which can trigger acroparesthesias, and use of spray bottles or cooling vests for hypohidrosis. Comprehensive management guidelines are available for pediatric and adult patients with Fabry disease $(63,68,69)$. 
At the time of this manuscript preparation, the Food and Drug Administration (FDA) approved migalastat $\left(\right.$ Galafold $\left.{ }^{\mathrm{TM}}\right)$ for the treatment of adults with Fabry disease in the United States. Migalastat is approved in the European Union, Australia, Canada, Israel, Japan, and other countries. Migalastat is a pharmacological chaperone that binds to the active site of the alpha-galactosidase enzyme and stabilizes the mutant protein. It is administered orally and may be an alternative treatment for Fabry patients who are unable to tolerate ERT due to antibody formation, infusion-related reactions, or other reasons. While the phase 3 clinical trial did not show a significant effect on GFR and left ventricular mass index (70), extension studies and long-term follow-up are needed to determine the clinical benefit of migalastat and its role in the treatment of Fabry disease.

\section{Acid sphingomyelinase deficiency}

Acid sphingomyelinase deficiency, formerly known as Niemann-Pick Disease type $\mathrm{A} / \mathrm{B}$, is a disorder of sphingolipid metabolism resulting in the accumulation of sphingomyelin in tissues throughout the body, in particular the spleen, liver, lungs, bone marrow, and in some cases brain. Historically, the disorder was classified into neuronopathic (Niemann-Pick Disease type A) and non-neuronopathic (Niemann-Pick Disease type B) forms, although they share a common metabolic defect. The disorder is now categorized into three phenotypes based on the presence of neurologic disease as well as age of onset and life expectancy (71). However, these classifications represent a single, broad continuum.

At the most severe end of the spectrum, the infantile, neurovisceral form of acid sphinogomyelinase deficiency presents in the first few months of life with failure to thrive and hepatosplenomegaly, which can be massive. Feeding difficulties, vomiting, diarrhea, cholestatic jaundice, and recurrent respiratory infections are also common. A macular cherry red spot can be seen in all infants by 12 months (72). Developmental stagnation followed by regression lead to death by 3 years (mean age 27 months) (71). Infantile neurovisceral acid sphinogomyelinase deficiency is common in the Ashkenazi Jewish population due to founder mutations in SMPD1.

At the mildest end of the disease spectrum, chronic visceral acid sphinogomyelinase deficiency can present from childhood to the fourth or fifth decade of life, with survival ranging from the second to eighth decade or later $(72,73)$. There is no neurologic involvement. Hepatosplenomegaly in childhood is often the first sign, although the diagnosis may not be apparent until adulthood. Pulmonary disease is present in $>80 \%$ of patients and is a major cause of morbidity and mortality (72). The deposition of sphingomyelin in the lungs causes both restrictive and interstitial lung disease, leading to exercise intolerance, recurrent pneumonias, and oxygen dependency (73). Cytopenias are common, in particular thrombocytopenia, which is seen in $>50 \%$ of patients (72). Dyslipidemia with low HDL, elevated LDL and elevated triglycerides is present in the majority of patients, and $10 \%$ have coronary artery disease or cardiac valve disease (72). Osteopenia and osteoporosis are common in adults.

An intermediate form, termed chronic neurovisceral acid sphingomyelinase deficiency, is characterized by the neurologic and somatic features of the other forms, but is less severe than infantile neurovisceral acid sphingomyelinase deficiency. The most common presenting sign is hepatosplenomegaly in childhood. The spleen can be 10-20 times the predicted size (73). Hepatic complications include transaminitis, fibrosis, and cirrhosis. Poor growth is common, and most children and adolescents are below average in height and weight. Development proceeds normally until age 2-7 years. Mild neurologic symptoms then appear, often manifesting as learning disabilities, hypotonia, and hyporeflexia. With time, ataxia, cognitive impairment, and loss of motor function follow. Age of death ranges from childhood to adulthood.

The diagnosis of acid sphingomyelinase deficiency is based on recognition of clinical features along with biochemical and genetic testing. Elevated plasma oxysterols, particularly lyso-sphingomyelin, is highly suggestive. Bone marrow biopsy demonstrates lipid-laden macrophages (foam cells). Deficiency of acid sphingomyelinase activity in lymphocytes or cultured fibroblasts along with identification of pathogenic variants in SMPD1 confirm the diagnosis.

There is currently no disease-modifying therapy for acid sphingomyelinase deficiency. At the time of this manuscript preparation, a phase $2 / 3$ clinical trial for ERT is being conducted (74). However, based on experience with other LSD's, ERT would likely not impact the CNS disease associated with acid sphingomyelinase deficiency (75).

\section{Other sphingolipidoses}

Tay-Sachs disease, Sandhoff disease, GM1 gangliosidosis, Krabbe disease, and metachromatic leukodystrophy are other disorders involved in sphingolipid metabolism. While 
each has unique features, the common and predominant feature is progressive neurodegeneration and premature death. Currently, no disease-specific treatments are available for these LSD's.

\section{Niemann-Pick disease type C}

Although historically linked by a common name, NiemannPick disease type $\mathrm{C}(\mathrm{NP}-\mathrm{C})$ and acid sphingomyelinase deficiency (previously known as Niemann-Pick disease type $\mathrm{A} / \mathrm{B}$ ) differ in their disease mechanism and pathogenesis and are therefore unrelated as far as the metabolic disturbance, although they share clinical features. NP-C is caused by mutations in either NPC1 or NPC2, which encode proteins involved in cholesterol and lipid trafficking within the cell. When this process is impaired, excess lipid storage occurs, most prominently in the liver, spleen, and CNS. There is considerable heterogeneity as far as clinical features and disease severity.

NP-C can present in the neonatal period with fetal hydrops and cholestatic jaundice. In severe cases, infiltrative pulmonary disease and liver failure lead to death within the first few months of life. Infants who survive this period have sustained hepatosplenomegaly and may show early signs of neurologic involvement such as hypotonia and developmental delay. Roughly $40 \%$ of NP-C patients are diagnosed before age 6 years, 21\% between $6-11$ years, and $39 \%$ at 12 years or later (76). In the classic childhood form, patients present between 2-6 years of age with hepatosplenomegaly and subtle neurologic features, such as behavior problems or clumsiness. With time, deficits become more apparent including developmental delay, gait problems, cerebellar ataxia, dysarthria, seizures, and the characteristic vertical supranuclear gaze palsy, which is present in virtually all NP-C patients. Initially, only vertical saccades are impaired, but with advanced disease, horizontal saccades are also affected. Seizures can be medically refractory. Approximately $50 \%$ of patients with childhood onset NP-C have gelastic cataplexy (77), which is sudden loss of muscle tone without loss of consciousness triggered by an emotional stimulus such as laughter. Children progressively deteriorate over time and generally do not survive beyond the second decade.

There is an inverse correlation between age of onset and rate of progression; the earlier the onset, the faster the disease progression. In addition, infants with NP-C tend to manifest with predominantly visceral signs and symptoms, whereas adolescents and adults more often exhibit neurologic and psychiatric problems, although hepatosplenomegaly is still fairly common $(77,78)$. The neurologic signs in adults are the same as those seen in children. Psychiatric symptoms, present in $40 \%$ of adolescents and adults, include hallucinations, delusions, cognitive decline, dementia, depression, bipolar disorder, disruptive and aggressive behavior $(77,78)$. Schizophrenialike psychosis may be resistant to treatment.

The first-line test in the diagnosis of NP-C is plasma oxysterol quantitation (79). C-triol in particular is a sensitive and specific biomarker for NP-C. However, oxysterols are elevated in other conditions, including acid sphingomyelinase deficiency. Ultimate confirmation of the diagnosis is made by genetic testing with identification of 2 pathogenic variants in NPC1 or NPC2. In the past, filipin staining on fibroblasts was considered the gold standard test for the diagnosis of NP-C, but this method has been superseded by the combination of biomarkers and molecular testing. In some instances, it is still used if DNA testing is equivocal.

As far as treatment, the medication miglustat $\left(\right.$ Zavesca $\left.^{\circledR}\right)$ is approved for NP-C in the European Union, Canada, Japan. Miglustat was originally developed for Gaucher disease (see above) and was approved in Europe for use in NP-C patients in 2009. At the time of this manuscript preparation, the FDA has not approved miglustat for the treatment of NP-C in the United States, but some health care providers are able to prescribe it with insurance authorization. In clinical trials, miglustat was shown to stabilize and/or improve neurologic function in children and adults with NP-C as measured by horizontal saccadic eye movement velocity, swallowing ability, and ambulation $(80,81)$. Subsequent studies also demonstrated improvement in dystonia, dysmetria, and dysarthria as well as slower rate of disease progression across all age groups $(82,83)$. Miglustat should be offered to all NP-C patients with evidence of neurologic, psychiatric, or cognitive impairment (84). For those in whom symptoms are not yet manifest, such as individuals diagnosed due to a positive family history, the timing of initiation of therapy should be discussed with the patient or parent/caregiver.

Management of NP-C is otherwise supportive and aimed at treating symptoms and improving quality of life. Tricyclic antidepressants and CNS stimulants can help in treating gelastic cataplexy (85). Comprehensive management guidelines are available for Niemann-Pick disease type C (84).

\section{Neuronal ceroid lipofuscinoses}

The neuronal ceroid lipofuscinoses are a group of 
Table 3 The neuronal ceroid lipofuscinoses, associated genes, phenotypes, and encoded protein

\begin{tabular}{|c|c|c|c|}
\hline Gene & Disease/phenotype & Alternative name & Encoded protein \\
\hline \multirow{3}{*}{ CLN1/PPT1 } & CLN1 disease, late infantile & \multirow{3}{*}{ Infantile } & \multirow{3}{*}{ Palmitoyl protein thioesterase 1, PPT1 } \\
\hline & CLN1 disease, juvenile & & \\
\hline & CLN1 disease, adult & & \\
\hline CLN2/TPP1 & CLN2 disease, juvenile & Late-Infantile & Tripeptidyl peptidase 1, TPP1 \\
\hline CLN3 & CLN3 disease & Juvenile & CLN3 transmembrane protein/Battenin \\
\hline \multirow[t]{2}{*}{ CLN4/DNAJC5 } & \multirow[t]{2}{*}{ CLN4 disease } & Adult autosomal dominant Batten & \multirow[t]{2}{*}{ Cysteine string protein a } \\
\hline & & Kuf's disease & \\
\hline CLN5 & CLN5 disease, adult & Finnish variant late - infantile & Ceroid-lipofuscinosis neuronal protein 5 \\
\hline \multirow[t]{2}{*}{ CLN6 } & $\begin{array}{l}\text { CLN6 disease, late infantile } \\
\text { CLN6 disease, juvenile }\end{array}$ & \multirow[t]{2}{*}{$\begin{array}{l}\text { Early juvenile variant or I ate infantile } \\
\text { variant }\end{array}$} & \multirow[t]{2}{*}{$\begin{array}{l}\text { Ceroid-lipofuscinosis neuronal } \\
\text { protein } 6\end{array}$} \\
\hline & CLN6 disease, adult & & \\
\hline CLN7/MFSD8 & CLN7 disease, late infantile & Late-infantile variant & $\begin{array}{l}\text { Major facilitator superfamily domain-- } \\
\text { containing protein } 8\end{array}$ \\
\hline \multirow[t]{2}{*}{ CLN8 } & \multirow[t]{2}{*}{ CLN8 disease, late infantile } & Late-infantile variant & \multirow[t]{2}{*}{ Protein CLN8 } \\
\hline & & $\begin{array}{l}\text { EPMR (progressive epilepsy with } \\
\text { mental retardation) }\end{array}$ & \\
\hline CLN11/GRN & CLN11 disease, adult & $\begin{array}{l}\text { Adult (heterozygous mutations } \\
\text { cause frontotemporal lobar dementia) }\end{array}$ & Progranulin \\
\hline CLN12/ATP13A2 & CLN12 disease, juvenile & $\begin{array}{l}\text { CLN12 disease } \\
\text { Juvenile (mutations also cause Kufor- } \\
\text { Rakeb syndrome) }\end{array}$ & ATPase type $13 \mathrm{~A} 2$ \\
\hline CLN13/CTSF & CLN13 disease, adult & Adult Kufs type B & Cathepsin F \\
\hline CLN14/KCTD7 & CLN14 disease, infantile & CLN14 disease, infantile & $\begin{array}{l}\text { Potassium channel tetramerization } \\
\text { domain containing protein } 7\end{array}$ \\
\hline
\end{tabular}

Adapted from Williams RE and Mole SE 2012 (86) with permission.

progressive, neurodegenerative disorders named for the histological appearance of storage material containing autofluorescent lipopigments. The term Batten disease has been applied collectively to this group of disorders, although more recent understanding of the molecular basis of disease has led to a system of nomenclature whereby subtypes are designated by a number based on the associated gene and phenotype, e.g., CLN2 (Table 3) (86). 
There are currently 13 genes known to cause neuronal ceroid lipofuscinosis. Most have an autosomal recessive inheritance pattern, but autosomal dominant inheritance can be seen in one of the adult-onset forms, CLN4. The mechanism of pathogenesis has been characterized for some disorders. For example, deficiency of the lysosomal enzyme palmitoyl-protein thioesterase 1 in the classic infantile form results in the inability to remove long-chain fatty acids from proteins, which impairs their normal breakdown. For other disorders, the pathogenesis remains unclear.

One of the most prevalent types of neuronal ceroid lipofuscinosis is CLN2, also known as the classic lateinfantile type although other presentations can occur (Table 3). Infants with CLN2 develop normally for the first year or two of life. Language delay then becomes apparent, and seizures, often the first sign of serious disease, begin around 3 years of age. Seizure semiology is varied and includes generalized tonic-clonic, partial, atonic, myoclonic, and status epilepticus. (87). Myoclonic jerks are observed in all patients by 3-4 years of age. Ataxia and developmental regression follow, with rapid loss of language and motor milestones. Children are nonverbal and non-ambulatory by 4-6 years $(87,88)$. Retinopathy and blindness also become apparent during this period, leading to total disability. Children rarely survive beyond early adolescence.

In addition to classic late-infantile neuronal ceroid lipofuscinosis, congenital, infantile, juvenile, and adultonset forms exist (Table 3). They share the common features of motor and cognitive decline, epilepsy, ataxia, and vision loss with variable rate of progression (89). In older patients, behavior problems, dementia, and psychiatric symptoms can be seen.

The neuronal ceroid lipofuscinosis can be diagnosed by demonstration of deficient enzyme activity in some cases (e.g., CLN, CLN2, etc.). In others, electron microscopy provides evidence of disease such as granular osmiophilic deposits, curvilinear profiles, and fingerprint profiles. Due to the genetic heterogeneity, the best method of diagnosis is a gene panel, which examines all of the possible genes at once and is readily available in commercial labs.

Currently, disease-specific treatment is only available for CLN2. Cerliponase alfa $\left(\right.$ Brineura $\left.^{\circledR}\right)$ is an intracerebroventricular ERT that was approved for the treatment of CLN2 in 2017. In clinical trials, cerliponase alfa, administered via a surgically implanted intraventricular access device, slowed the loss of ambulation. The mean rate of functional decline in treated patients as measured by a standardized assessment tool was 0.43 units over
48 weeks compared to 2.09 units in the untreated group (90). Cerliponase alfa is approved in the United States for children $\geq 3$ years and in the European Union in all age groups. Clinical trials to determine long-term efficacy are ongoing. Other aspects of care, including nutrition, therapies, seizure management, and palliative care, are summarized by Williams et al. (91).

\section{Concluding thoughts}

The LSDs are a heterogeneous group of disorders that are individually rare but collectively common. While clinical features vary amongst the disorders, they all share a progressive course. Treatment is available for some, but significant morbidity and mortality remain. The development of novel therapies and the inclusion of LSD's on newborn screening panels will further change the natural history of these disorders.

\section{Acknowledgements}

None.

\section{Footnote}

Conflicts of Interest: The author has no conflicts of interest to declare.

\section{References}

1. Shapiro EG, Nestrasil I, Rudser K, et al. Neurocognition across the spectrum of mucopolysaccharidosis type I: Age, severity, and treatment. Mol Genet Metab 2015;116:61-8.

2. Palmucci S, Attina G, Lanza ML, et al. Imaging findings of mucopolysaccharidoses: a pictorial review. Insights Imaging 2013;4:443-59.

3. Bertola F, Filocamo M, Casati G, et al. IDUA mutational profiling of a cohort of 102 European patients with mucopolysaccharidosis type I: identification and characterization of 35 novel alpha-L-iduronidase (IDUA) alleles. Hum Mutat 2011;32:E2189-210.

4. Aldenhoven M, Wynn RF, Orchard PJ, et al. Long-term outcome of Hurler syndrome patients after hematopoietic cell transplantation: an international multicenter study. Blood 2015;125:2164-72.

5. Krivit W, Sung JH, Shapiro EG, et al. Microglia: the effector cell for reconstitution of the central nervous system following bone marrow transplantation for 
lysosomal and peroxisomal storage diseases. Cell

Transplant 1995;4:385-92.

6. Guffon N, Bertrand Y, Forest I, et al. Bone marrow transplantation in children with Hunter syndrome: outcome after 7 to 17 years. J Pediatr 2009;154:733-7.

7. Kubaski F, Yabe H, Suzuki Y, et al. Hematopoietic Stem Cell Transplantation for Patients with

Mucopolysaccharidosis II. Biol Blood Marrow Transplant 2017;23:1795-803.

8. Selvanathan A, Ellaway C, Wilson C, et al.

Effectiveness of Early Hematopoietic Stem Cell Transplantation in Preventing Neurocognitive Decline in Mucopolysaccharidosis Type II: A Case Series. JIMD Rep 2018;41:81-9.

9. Martins AM, Dualibi AP, Norato D, et al. Guidelines for the management of mucopolysaccharidosis type I. J Pediatr 2009;155:S32-46.

10. Scarpa M, Almassy Z, Beck M, et al. Mucopolysaccharidosis type II: European recommendations for the diagnosis and multidisciplinary management of a rare disease. Orphanet J Rare Dis 2011;6:72.

11. Valstar MJ, Neijs S, Bruggenwirth HT, et al. Mucopolysaccharidosis type IIIA: clinical spectrum and genotype-phenotype correlations. Ann Neurol 2010;68:876-87.

12. Shapiro EG, Nestrasil I, Delaney KA, et al. A Prospective Natural History Study of Mucopolysaccharidosis Type IIIA. J Pediatr 2016;170:278-87.e1-4.

13. Tchan MC, Sillence D. Extrapyramidal symptoms and medication use in Mucopolysaccharidosis type III. J Intellect Dev Disabil 2009;34:275-9.

14. Fraser J, Wraith JE, Delatycki MB. Sleep disturbance in mucopolysaccharidosis type III (Sanfilippo syndrome): a survey of managing clinicians. Clin Genet 2002;62:418-21.

15. Jones SA, Breen C, Heap F, et al. A phase $1 / 2$ study of intrathecal heparan- $\mathrm{N}$-sulfatase in patients with mucopolysaccharidosis IIIA. Mol Genet Metab 2016;118:198-205.

16. Marshall NR, Hassiotis S, King B, et al. Delivery of therapeutic protein for prevention of neurodegenerative changes: comparison of different CSF-delivery methods. Exp Neurol 2015;263:79-90.

17. Montano AM, Tomatsu S, Brusius A, et al. Growth charts for patients affected with Morquio A disease. Am J Med Genet A 2008;146A:1286-95.

18. Hendriksz CJ, Harmatz P, Beck M, et al. Review of clinical presentation and diagnosis of mucopolysaccharidosis IVA. Mol Genet Metab 2013;110:54-64.
19. Hendriksz CJ, Berger KI, Giugliani R, et al. International guidelines for the management and treatment of Morquio A syndrome. Am J Med Genet A 2015;167a:11-25.

20. Hendriksz CJ, Burton B, Fleming TR, et al. Efficacy and safety of enzyme replacement therapy with BMN 110 (elosulfase alfa) for Morquio A syndrome (mucopolysaccharidosis IVA): a phase 3 randomised placebocontrolled study. J Inherit Metab Dis 2014;37:979-90.

21. Muenzer J. Overview of the mucopolysaccharidoses. Rheumatology (Oxford) 2011;50 Suppl 5:v4-12.

22. Harmatz P, Hendriksz CJ, Lampe C, et al. The effect of galsulfase enzyme replacement therapy on the growth of patients with mucopolysaccharidosis VI (Maroteaux-Lamy syndrome). Mol Genet Metab 2017;122:107-12.

23. Braunlin E, Rosenfeld H, Kampmann C, et al. Enzyme replacement therapy for mucopolysaccharidosis VI: longterm cardiac effects of galsulfase (Naglazyme(R)) therapy. J Inherit Metab Dis 2013;36:385-94.

24. Furujo M, Kosuga M, Okuyama T. Enzyme replacement therapy attenuates disease progression in two Japanese siblings with mucopolysaccharidosis type VI: 10-Year follow up. Mol Genet Metab Rep 2017;13:69-75.

25. Harmatz P, Shediac R. Mucopolysaccharidosis VI: pathophysiology, diagnosis and treatment. Front Biosci (Landmark Ed) 2017;22:385-406.

26. Montano AM, Lock-Hock N, Steiner RD, et al. Clinical course of sly syndrome (mucopolysaccharidosis type VII). J Med Genet 2016;53:403-18.

27. Zielonka M, Garbade SF, Kolker S, et al. Quantitative clinical characteristics of 53 patients with MPS VII: a cross-sectional analysis. Genet Med 2017;19:983-8.

28. Byrne BJ, Kishnani PS, Case LE, et al. Pompe disease: design, methodology, and early findings from the Pompe Registry. Mol Genet Metab 2011;103:1-11.

29. van Capelle CI, Poelman E, Frohn-Mulder IM, et al. Cardiac outcome in classic infantile Pompe disease after 13 years of treatment with recombinant human acid alphaglucosidase. Int J Cardiol 2018;269:104-10.

30. Kishnani PS, Corzo D, Nicolino M, et al. Recombinant human acid [alpha]-glucosidase: major clinical benefits in infantile-onset Pompe disease. Neurology 2007;68:99-109.

31. Levine JC, Kishnani PS, Chen YT, et al. Cardiac remodeling after enzyme replacement therapy with acid alpha-glucosidase for infants with Pompe disease. Pediatr Cardiol 2008;29:1033-42.

32. McDowell R, Li JS, Benjamin DK Jr, et al. Arrhythmias in patients receiving enzyme replacement therapy for infantile Pompe disease. Genet Med 2008;10:758-62. 
33. Prater SN, Banugaria SG, DeArmey SM, et al. The emerging phenotype of long-term survivors with infantile Pompe disease. Genet Med 2012;14:800-10.

34. Schoser B, Stewart A, Kanters S, et al. Survival and longterm outcomes in late-onset Pompe disease following alglucosidase alfa treatment: a systematic review and metaanalysis. J Neurol 2017;264:621-30.

35. Kronn DF, Day-Salvatore D, Hwu WL, et al. Management of Confirmed Newborn-Screened Patients With Pompe Disease Across the Disease Spectrum. Pediatrics 2017;140:S24-45.

36. Kishnani PS, Steiner RD, Bali D, et al. Pompe disease diagnosis and management guideline. Genet Med 2006;8:267-88.

37. Tarnopolsky M, Katzberg H, Petrof BJ, et al. Pompe Disease: Diagnosis and Management. Evidence-Based Guidelines from a Canadian Expert Panel. Can J Neurol Sci 2016;43:472-85.

38. Cupler EJ, Berger KI, Leshner RT, et al. Consensus treatment recommendations for late-onset Pompe disease. Muscle Nerve 2012;45:319-33.

39. Mehta A. Epidemiology and natural history of Gaucher's disease. Eur J Intern Med 2006;17 Suppl:S2-5.

40. Charrow J, Andersson HC, Kaplan P, et al. The Gaucher registry: demographics and disease characteristics of 1698 patients with Gaucher disease. Arch Intern Med 2000;160:2835-43.

41. Zimran A, Belmatoug N, Bembi B, et al. Demographics and patient characteristics of 1209 patients with Gaucher disease: Descriptive analysis from the Gaucher Outcome Survey (GOS). Am J Hematol 2018;93:205-12.

42. Koprivica V, Stone DL, Park JK, et al. Analysis and classification of 304 mutant alleles in patients with type 1 and type 3 Gaucher disease. Am J Hum Genet 2000;66:1777-86.

43. Weiss K, Gonzalez A, Lopez G, et al. The clinical management of Type 2 Gaucher disease. Mol Genet Metab 2015;114:110-22.

44. Charrow J, Andersson HC, Kaplan P, et al. Enzyme replacement therapy and monitoring for children with type 1 Gaucher disease: consensus recommendations. J Pediatr 2004;144:112-20.

45. Kaplan P, Baris H, De Meirleir L, et al. Revised recommendations for the management of Gaucher disease in children. Eur J Pediatr 2013;172:447-58.

46. Zimran A, Wajnrajch M, Hernandez B, et al. Taliglucerase alfa: safety and efficacy across 6 clinical studies in adults and children with Gaucher disease. Orphanet J Rare Dis
2018;13:36.

47. Weinreb NJ, Charrow J, Andersson HC, et al. Effectiveness of enzyme replacement therapy in 1028 patients with type 1 Gaucher disease after 2 to 5 years of treatment: a report from the Gaucher Registry. Am J Med 2002;113:112-9.

48. Weinreb NJ, Goldblatt J, Villalobos J, et al. Long-term clinical outcomes in type 1 Gaucher disease following 10 years of imiglucerase treatment. J Inherit Metab Dis 2013;36:543-53.

49. Zimran A, Wang N, Ogg C, et al. Seven-year safety and efficacy with velaglucerase alfa for treatment-naive adult patients with type 1 Gaucher disease. Am J Hematol 2015;90:577-83.

50. Smith L, Rhead W, Charrow J, et al. Long-term velaglucerase alfa treatment in children with Gaucher disease type 1 naive to enzyme replacement therapy or previously treated with imiglucerase. Mol Genet Metab 2016;117:164-71.

51. Elstein D, Hollak C, Aerts JM, et al. Sustained therapeutic effects of oral miglustat (Zavesca, N-butyldeoxynojirimycin, OGT 918) in type I Gaucher disease. J Inherit Metab Dis 2004;27:757-66.

52. Mistry PK, Lukina E, Ben Turkia H, et al. Outcomes after 18 months of eliglustat therapy in treatment-naive adults with Gaucher disease type 1: The phase 3 ENGAGE trial. Am J Hematol 2017;92:1170-6.

53. Cohen IJ, Kornreich L, Mekhmandarov S, et al. Effective treatment of painful bone crises in type I gaucher's disease with high dose prednisolone. Arch Dis Child 1996;75:218-22.

54. Pastores GM, Weinreb NJ, Aerts H, et al. Therapeutic goals in the treatment of Gaucher disease. Semin Hematol 2004;41:4-14.

55. Biegstraaten M, Cox TM, Belmatoug N, et al. Management goals for type 1 Gaucher disease: An expert consensus document from the European working group on Gaucher disease. Blood Cells Mol Dis 2018;68:203-8.

56. El-Beshlawy A, Tylki-Szymanska A, Vellodi A, et al. Long-term hematological, visceral, and growth outcomes in children with Gaucher disease type 3 treated with imiglucerase in the International Collaborative Gaucher Group Gaucher Registry. Mol Genet Metab 2017;120:47-56.

57. Schiffmann R, Fitzgibbon EJ, Harris C, et al. Randomized, controlled trial of miglustat in Gaucher's disease type 3. Ann Neurol 2008;64:514-22.

58. Hopkin RJ, Bissler J, Banikazemi M, et al. Characterization of Fabry disease in 352 pediatric patients in the Fabry Registry. Pediatr Res 2008;64:550-5. 
59. Mehta A, Ricci R, Widmer U, et al. Fabry disease defined: baseline clinical manifestations of 366 patients in the Fabry Outcome Survey. Eur J Clin Invest 2004;34:236-42.

60. Schaefer E, Mehta A, Gal A. Genotype and phenotype in Fabry disease: analysis of the Fabry Outcome Survey. Acta Paediatr Suppl 2005;94:87-92; discussion 79.

61. Oqvist B, Brenner BM, Oliveira JP, et al. Nephropathy in Fabry disease: the importance of early diagnosis and testing in high-risk populations. Nephrol Dial Transplant 2009;24:1736-43.

62. Branton MH, Schiffmann R, Sabnis SG, et al. Natural history of Fabry renal disease: influence of alphagalactosidase A activity and genetic mutations on clinical course. Medicine (Baltimore) 2002;81:122-38.

63. Ortiz A, Germain DP, Desnick RJ, et al. Fabry disease revisited: Management and treatment recommendations for adult patients. Mol Genet Metab 2018;123:416-27.

64. Wilcox WR, Oliveira JP, Hopkin RJ, et al. Females with Fabry disease frequently have major organ involvement: lessons from the Fabry Registry. Mol Genet Metab 2008;93:112-28.

65. Deegan PB, Baehner AF, Barba Romero MA, et al. Natural history of Fabry disease in females in the Fabry Outcome Survey. J Med Genet 2006;43:347-52.

66. Rombach SM, Smid BE, Linthorst GE, et al. Natural course of Fabry disease and the effectiveness of enzyme replacement therapy: a systematic review and metaanalysis: effectiveness of ERT in different disease stages. J Inherit Metab Dis 2014;37:341-52.

67. Anderson LJ, Wyatt KM, Henley W, et al. Long-term effectiveness of enzyme replacement therapy in Fabry disease: results from the NCS-LSD cohort study. J Inherit Metab Dis 2014;37:969-78.

68. Hopkin RJ, Jefferies JL, Laney DA, et al. The management and treatment of children with Fabry disease: A United States-based perspective. Mol Genet Metab 2016;117:104-13.

69. Wanner C, Arad M, Baron R, et al. European expert consensus statement on therapeutic goals in Fabry disease. Mol Genet Metab 2018;124:189-203.

70. Germain DP, Hughes DA, Nicholls K, et al. Treatment of Fabry's Disease with the Pharmacologic Chaperone Migalastat. N Engl J Med 2016;375:545-55.

71. McGovern MM, Dionisi-Vici C, Giugliani R, et al. Consensus recommendation for a diagnostic guideline for acid sphingomyelinase deficiency. Genet Med 2017;19:967-74.

72. McGovern MM, Avetisyan R, Sanson BJ, et al. Disease manifestations and burden of illness in patients with acid sphingomyelinase deficiency (ASMD). Orphanet J Rare Dis 2017;12:41.

73. McGovern MM, Wasserstein MP, Giugliani R, et al. A prospective, cross-sectional survey study of the natural history of Niemann-Pick disease type B. Pediatrics 2008;122:e341-9.

74. Wasserstein MP, Jones SA, Soran H, et al. Successful within-patient dose escalation of olipudase alfa in acid sphingomyelinase deficiency. Mol Genet Metab 2015;116:88-97.

75. Schuchman EH, Desnick RJ. Types A and B NiemannPick disease. Mol Genet Metab 2017;120:27-33.

76. Wraith JE, Guffon N, Rohrbach M, et al. Natural history of Niemann-Pick disease type $\mathrm{C}$ in a multicentre observational retrospective cohort study. Mol Genet Metab 2009;98:250-4.

77. Patterson MC, Mengel E, Wijburg FA, et al. Disease and patient characteristics in NP-C patients: findings from an international disease registry. Orphanet J Rare Dis $2013 ; 8: 12$.

78. Wraith JE, Sedel F, Pineda M, et al. Niemann-Pick type C Suspicion Index tool: analyses by age and association of manifestations. J Inherit Metab Dis 2014;37:93-101.

79. Patterson MC, Clayton P, Gissen P, et al. Recommendations for the detection and diagnosis of Niemann-Pick disease type C: An update. Neurol Clin Pract 2017;7:499-511.

80. Patterson MC, Vecchio D, Prady H, et al. Miglustat for treatment of Niemann-Pick C disease: a randomised controlled study. Lancet Neurol 2007;6:765-72.

81. Patterson MC, Vecchio D, Jacklin E, et al. Long-term miglustat therapy in children with Niemann-Pick disease type C. J Child Neurol 2010;25:300-5.

82. Patterson MC, Mengel E, Vanier MT, et al. Stable or improved neurological manifestations during miglustat therapy in patients from the international disease registry for Niemann-Pick disease type C: an observational cohort study. Orphanet J Rare Dis 2015;10:65.

83. Pineda M, Wraith JE, Mengel E, et al. Miglustat in patients with Niemann-Pick disease Type C (NP-C): a multicenter observational retrospective cohort study. Mol Genet Metab 2009;98:243-9.

84. Patterson MC, Hendriksz CJ, Walterfang M, et al. Recommendations for the diagnosis and management of Niemann-Pick disease type C: an update. Mol Genet Metab 2012;106:330-44.

85. Philippart M, Engel J Jr, Zimmerman EG. Gelastic 
cataplexy in Niemann-Pick disease group C and related variants without generalized sphingomyelinase deficiency. Ann Neurol 1983;14:492-3.

86. Williams RE, Mole SE. New nomenclature and classification scheme for the neuronal ceroid lipofuscinoses. Neurology 2012;79:183-91.

87. Perez-Poyato MS, Marfa MP, Abizanda IF, et al. Late infantile neuronal ceroid lipofuscinosis: mutations in the CLN2 gene and clinical course in Spanish patients. J Child Neurol 2013;28:470-8.

Cite this article as: Sun A. Lysosomal storage disease overview. Ann Transl Med 2018;6(24):476. doi: 10.21037/atm.2018.11.39
88. Worgall S, Kekatpure MV, Heier L, et al. Neurological deterioration in late infantile neuronal ceroid lipofuscinosis. Neurology 2007;69:521-35.

89. Nita DA, Mole SE, Minassian BA. Neuronal ceroid lipofuscinoses. Epileptic Disord 2016;18:73-88.

90. Markham A. Cerliponase Alfa: First Global Approval. Drugs 2017;77:1247-9.

91. Williams RE, Adams HR, Blohm M, et al. Management Strategies for CLN2 Disease. Pediatr Neurol 2017;69:102-12. 